\title{
BMJ Open Longitudinal study on the effect of surgical weight loss on beat-to-beat blood pressure variability in patients undergoing bariatric surgery: a study protocol
}

\author{
Souha Fares, ${ }^{1}$ Nour-Mounira Z Bakkar (D) , ${ }^{1}$ Ramzi Alami, ${ }^{2}$ Issam Lakkis, ${ }^{3}$ \\ Kamal Badr ${ }^{4}$
}

To cite: Fares S, Bakkar N-MZ, Alami R, et al. Longitudinal study on the effect of surgical weight loss on beat-to-beat blood pressure variability in patients undergoing bariatric surgery: a study protocol. BMJ Open 2021;11:e050957. doi:10.1136/ bmjopen-2021-050957

- Prepublication history for this paper is available online. To view these files, please visit the journal online (http://dx.doi. org/10.1136/bmjopen-2021050957).

Received 08 March 2021 Accepted 30 September 2021

Check for updates

(C) Author(s) (or their employer(s)) 2021. Re-use permitted under CC BY-NC. No commercial re-use. See rights and permissions. Published by BMJ.

${ }^{1}$ Rafic Hariri School of Nursing, American University of Beirut,

Beirut, Lebanon

${ }^{2}$ Department of Surgery, American University of Beirut Medical Center, Beirut, Lebanon ${ }^{3}$ Department of Mechanical Engineering, American University of Beirut Faculty of Engineering and Architecture, Beirut, Lebanon

${ }^{4}$ Department of Internal Medicine, American University of Beirut Faculty of Medicine, Beirut, Lebanon

Correspondence to

Dr Souha Fares;

sf31@aub.edu.lb

\section{ABSTRACT}

Introduction Alterations in linear and non-linear parameters of beat-to-beat blood pressure variability (BPV) have been shown to predict disease prognosis and distinguish between risk categories in various pathological conditions, independently of average blood pressure levels. Obesity places subjects at elevated risk of vascular diseases, including hypertension, resulting in serious cardiac, respiratory and cerebral events. However, little is known about the status of vascular dynamics in obese and morbidly obese adults.

Methods and analysis In this present quasi-experimental longitudinal study, changes in beat-to-beat BPV, using continuous, non-invasive blood pressure monitoring, in obese subjects undergoing bariatric surgery are characterised. The capacity of linear and non-linear measures of BPV to detect differences between hypertensive, prehypertensive and normotensive obese subjects prebariatric and postbariatric surgery are tested. Additionally, potential correlations between beat-to-beat BPV and age, body mass index, gender and comorbidities will be investigated. In parallel, the impact of the unsteady fluctuations of beat-to-beat blood pressure on the dynamic stresses imparted by blood flow on blood vessel walls will be explored. We expect to find altered BPV profiles in hypertensive and prehypertensive subjects as compared with normotensive subjects. We also expect to see differential normalisation in BPV profiles between hypertensive, prehypertensive and normotensive subjects over time.

Ethics and dissemination The study has been approved by the Institutional Review Board at the American University of Beirut (IRB ID: BI0-2018-0040). Study results will be made available to the public through publications in peer-reviewed journals and conference papers and/or presentations.

\section{INTRODUCTION}

Obese individuals are at increased risk of cardiovascular morbidity and mortality. ${ }^{1}$ Indeed, weight gain has been associated with atherosclerosis, hypertension $(\mathrm{HTN})^{2}$ and
Strengths and limitations of this study

- This is a longitudinal study which accounts for intersubject differences and characteristics impacting beat-to-beat blood pressure variability (BPV).

- We use beat-to-beat BPV which allows for assessment of short-term changes mainly driven by autonomic control and vascular function reflecting in intraindividual differences.

- We employ both linear and non-linear parameters of beat-to-beat BPV which permits the characterisation of different profiles of BP fluctuations with different sensitivities and physiological determinants and/or correlates.

- One of the challenges we face during data collection is the ability to maintain the subject in an awake, yet motionless, state for $30 \mathrm{~min}$, to avoid interference of sleep or movement on beat-to-beat BPV.

vascular complications, that insidiously lead to cerebrovascular and respiratory vascular complications, including stroke and obstructive sleep apnoea (OSA) ${ }^{3-5}$ Weight loss strategies ideally aim at reducing disease states associated with excess body fat. One of these strategies, bariatric surgery, was shown to have long-term positive outcomes on disease prognosis and complications. ${ }^{6-10}$

While increased average blood pressure (BP) has been established to be associated with deleterious effects, BP variability (BPV) has recently gained insight as a potential underlying mechanism for end-organ damage. $\mathrm{BP}$ varies on the long, short and very short term. Changes in BP can be observed from week-to-week, day-to-day and even from beatto-beat. ${ }^{11}$ The degree of change in BP values can be quantified using linear (conventional) and/or non-linear variability indices. Linear parameters quantify the degree of dispersion 
in BP fluctuations and include the SD and coefficient of variation (CV); these have been shown to increase abnormally in different disease states. Non-linear parameters describe the complexity and degree of correlation in a time series ${ }^{12} 13$ and include complexity analysis and detrended fluctuation analysis (DFA). Higher complexity is physiologically desirable. ${ }^{14-17}$ Interestingly, studies have revealed the capacity of beat-to-beat BPV to detect premature changes in vascular structure and function, independently of average BP. ${ }^{18-22}$ Additionally, researchers have been interested in studying the relative powers of linear and non-linear parameters in identifying pathological mechanisms and discriminating among groups with progressive vascular diseases. ${ }^{141623}$

Characterisation of beat-to-beat BPV with respect to the status of blood vessels and end-organ damage in obese individuals, with or without concomitant HTN, remain scarce. The available studies investigate the status of beat-to-beat BPV in obese children in an attempt to study the early changes in vascular dynamics associated with obesity. ${ }^{24}{ }^{25}$ Whereas the results of one of the studies reflect a relatively intact pattern of beat-to-beat BPV in obese children, ${ }^{24}$ results from another demonstrate early detrimental autonomic processes. ${ }^{25}$ Moreover, adult subjects with abdominal obesity-associated HTN demonstrated an increase in daytime and night BPV positively correlated with body mass index (BMI) ${ }^{26}$ However, these results were only published as an abstract. Additionally, while the ameliorative effect of bariatric surgery has been previously demonstrated on heart rate (HR) variability in a patient population of obese diabetics, ${ }^{27} 28$ the responsiveness of beat-to-beat BP dynamics to metabolic improvements associated with bariatric surgery remains ill defined.

\section{Rationale}

In this longitudinal study, the aim is to describe the profile of beat-to-beat time series in obese and morbidly obese patients undergoing elective bariatric surgery. Particularly, the relative capacity of linear and non-linear variability parameters to differentiate among hypertensive, prehypertensive and non-hypertensive obese patients preoperation will be tested. Additionally, the responsiveness of beat-to-beat BP dynamics to surgical weight loss-induced metabolic amelioration over time will be studied. The potential outcome of bariatric surgery on beat-to-beat BP dynamics will be investigated. In parallel, the impact of unsteady BP dynamics on the normal and shear stresses imparted by blood flow on the vascular wall will be characterised to identify possible underlying endorgan damage related to increased vascular injury.

\section{Objectives}

1. To determine the longitudinal effect of weight loss through bariatric surgery on BP complexity and fluctuation measures among no-HTN, pre-HTN and HTN subjects over time over time (from preoperation to 3, 6 and 12 months postoperation).
2. To compare BPV metrics among no-HTN, pre-HTN and HTN subjects.

3. To assess the use of the new BPV metrics (complexity and DFA) alone or in combination with conventional BPV measures for discriminating between no-HTN and HTN patients.

4. To quantify the impact of the unsteady dynamics of the observed beat-to-beat pressure on the dynamic stresses imparted by the blood flow on the walls of the blood vessels

\section{METHODS AND ANALYSIS}

\section{Study design}

This is a single-centred, longitudinal, quasi-experimental study taking place at the American University of Beirut Medical Center (AUBMC), Lebanon in the Metabolic and Bariatric Unit.

\section{Participants}

The target population is obese and morbidly obese (BMI $\geq 30$ ) subjects who are scheduled for bariatric surgery (Laparoscopic Sleeve Gastrectomy or Roux-en-Y Gastric Bypass) at the AUBMC.

\section{Recruitment methods and study procedures}

Candidates for bariatric surgery are first approached and introduced to the study by their treating physician and then by the research assistant on their preoperation visit. Consent is then obtained. During this visit, beat-to-beat $\mathrm{BP}$ is monitored. Later, BP monitoring sessions take place at 3, 6 and 12 months postbariatric surgery, during postoperation follow-up clinic appointments (figure 1).

\section{Preoperation and postoperation beat-to-beat BPV monitoring} After their preoperation appointment, patients are seated in a comfortable chair next to a desk. Based on the patient's choice among a number of relaxing videos, a 30 min video is played on a desktop screen facing the patient. Continuous BP monitoring is carried out by plethysmography using a FINAPRES NOVA finometer (Finapres Medical Systems, Amsterdam, the Netherlands). The finger cuff is fitted based on a finger circumference ruler provided by the manufacturer. Notably, the finger cuff comes in three sizes: small, medium and large. For $30 \mathrm{~min}$, the subject is asked to remain quiet and abstain from moving. $5 \mathrm{~min}$ after the start of beat-to-beat BP monitoring, after a stable signal is obtained, brachial calibration is done using an arm cuff (sphygmomanometer) as per the manufacturer's recommendation. Finger BP signal is calibrated according to the average of two acquired brachial $\mathrm{BP}$ values to obtain reconstructed $\mathrm{BP}$ values. After this time, $\mathrm{BP}$ monitoring is paused for $1 \mathrm{~min}$ at 10 and $20 \mathrm{~min}$ to allow the finger to rest from continuous pressure. This process is repeated at three time points, which are 3, 6 and 12 months after the bariatric surgery.

\section{Inclusion and exclusion criteria}

All patients 18 years old and above who are scheduled for bariatric surgery at AUBMC will be approached for 


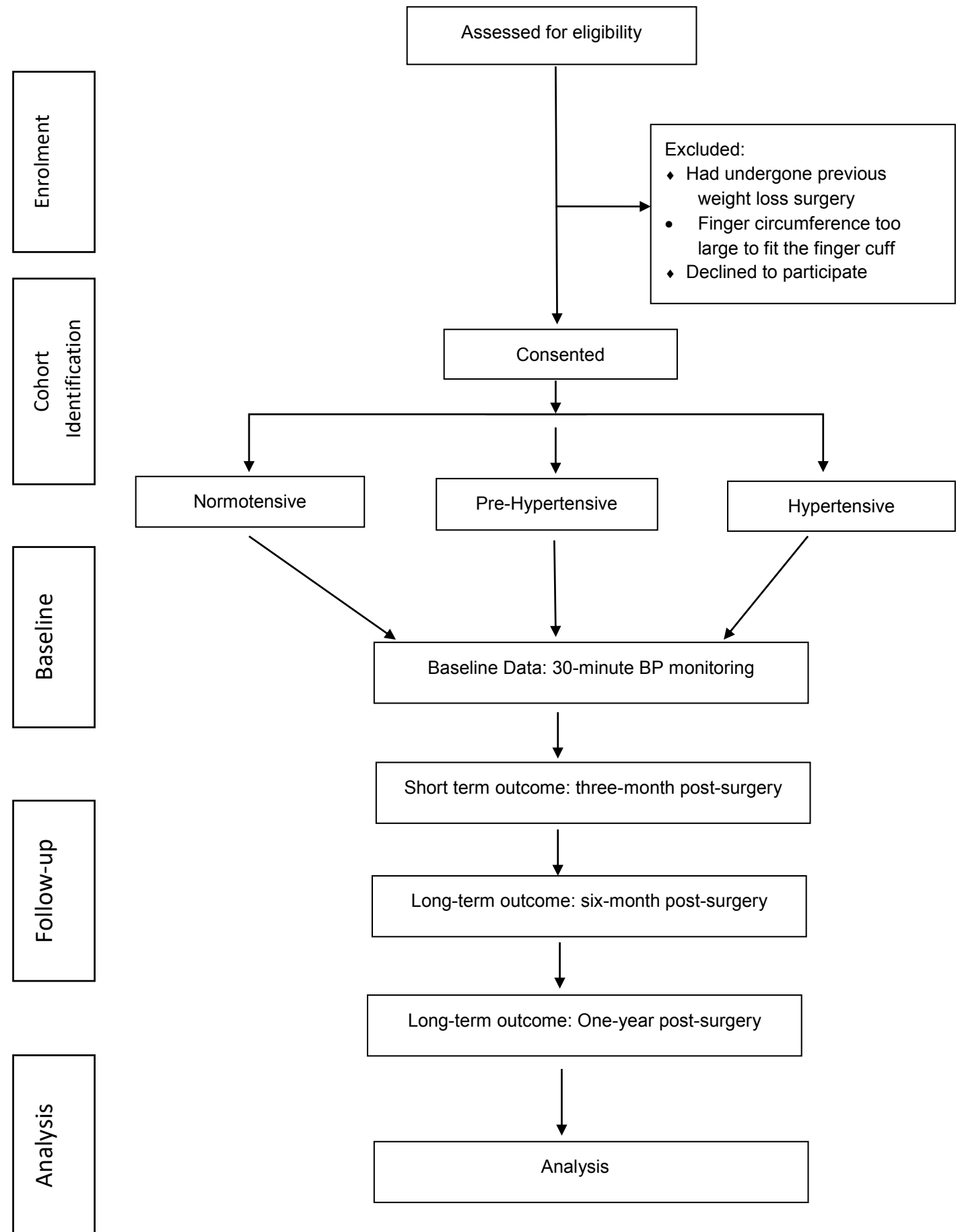

Figure 1 Flow of participants diagram which illustrates participants enrolment throughout the study.

this study. HTN, pre-HTN and normotensive subjects will be approached; HTN status is previously confirmed by the referring physician and will be retrieved from the patients' medical records. To avoid confounding factors and false negatives, patients who had undergone previous weight loss surgeries are excluded. Additionally, those whose arm or finger circumference is/are too large to fit the arm or finger cuffs available at the company are also excluded from the study.

\section{Measures}

\section{Outcomes}

The study outcomes are the variability parameters derived from the beat-to-beat BP time series described in the Plan of analysis section.

\section{Other variables}

From the beat-to-beat time series, the following measures will be derived: Systolic BP, diastolic BP, mean arterial 
pressure and HR. We will also collect other variables including: Sociodemographics: age (in years), weight (in $\mathrm{kg}$ ), gender, education (none, high school or less, college), marital status (single, married, divorced, widowed); life-style habits: Cigarette smoking (yes, no), narguile smoking (yes, no), alcohol consumption (yes, no), exercise (yes, no); Patient history of: heart disease, dyslipidaemia, diabetes, kidney, thyroid, OSA (yes, no); Family history of: heart disease, HTN, diabetes (yes, no) and classes of antihypertensive medication, if any.

\section{Sample size calculation}

We based our power analysis on the aim of determining the effect of weight loss through bariatric surgery on BP complexity and fluctuation measures among no-HTN, pre-HTN and HTN subjects. Since we found no previous studies similar to ours, we computed effect sizes based on available studies comparing entropy measures and DFA exponent between independent/dependent groups. Those effect sizes were moderate to high ( $\mathrm{f}=0.20-0.75$ in independent samples ${ }^{142930}$ ). To remain on the conservative side, we assumed a moderate effect size $(\mathrm{f}=0.25)$ in our sample size calculation. Power analysis for a betweengroup repeated measures design indicate that with three time point comparison to baseline ( 3 months, 6 months and 12 months), a sample size of 102 patients would have $80 \%$ power to detect a medium effect size of $25 \%$ at $\alpha=0.05$, assuming a medium correlation between the repeated measures $\rho=0.5$. Since this a longitudinal study, we assumed a $20 \%$ attrition rate, therefore, we aim to recruit a total of 129 subjects (43 in every group). The average yearly number of bariatric surgeries at AUBMC is around 231. GPower V.3.0.10 was used for sample size determination.

\section{Plan of analysis}

Conventional/linear variability analysis

$\mathrm{SD}$ and $\mathrm{CV}$ will be calculated.

\section{Complexity analysis}

Complexity analysis consists of measures that quantify the irregularity of biological signals, called entropy measures. Sample entropy (SampEn) is a measure of the self-similarity in a time series. It depends on two parameters: a dimension $m$ which is the number of consecutive data points in a pattern and a tolerance $r$ within which the $m$ points are considered a self-match. SampEn is the negative natural logarithm of the conditional probability that sequences within $r$ for the $m$ consecutive data points remain within $r$ for the next point. SampEn is related to approximate entropy (ApEn), but unlike it, SampEn eliminates self-counting of matches and as a result minimises the dependency of this complexity index on the length of the time series. Using SampEn will thus remove this bias introduced by ApEn. A Matlab code to compute SampEn, available on Physionet (http://www.physionet.org/physiotools/sampen/c/) was used in our previous study. ${ }^{31}$
Detrended fluctuation analysis

DFA has been developed to detect the correlations of varying ranges (short and long range) embedded in a time series using the fractal property. As used previously, DFA can be analysed in two ways: a single slope $\alpha$ for the whole time series, and double slopes, one for short-term correlations $\left(\alpha_{\mathrm{S}}\right)$ and one for long-term correlation $\left(\alpha_{\mathrm{L}}\right)$ based on an a priori cut-off $(\mathrm{n}=10)$. DFA scaling exponents $\alpha, \alpha_{S}$ and $\alpha_{L}$ are measures of the smoothness of a time series, higher correlations indicate increased predictability or smoother series. A Matlab (MathWorks, Natick, Massachusetts, USA) code to derive $\alpha, \alpha_{\mathrm{S}}$ and $\alpha_{\mathrm{L}}$ was implemented by the research team and used in our previous study. ${ }^{31}$

\section{Quantification of haemodynamic forces (aim 4)}

The dynamic forces imparted by the blood flow on the blood vessels caused by the mechanical loading- the observed beat-to-beat BP- will be quantified using a physically based computational model. This will be accomplished as follows. A model construction tool (Simpleware) will be used to construct a 3D model form medical images (CT Scans, MRI, etc). A computational mesh is then generated from the geometrical model. The blood flow will then be dynamically simulated in space (3D) and time using a physically based computational tools (OpenFOAM or ANSYS) that numerically solve the system of coupled partial differential equations governing the fluid/solid continuum behaviour. The observed (or properly scaled) beat-to-beat pressure history will act as the forcing to the problem. In addition to the mesh and the forcing, well-established stress/strain and stress/rate of strain models for the tissue and blood will be employed.

\section{Statistical analysis}

We will perform descriptive statistics to determine and compare the subject group characteristics including demographics and medical history. For aim 1, we will use linear mixed models to assess the changes in the variability metrics (as determined by SD, CV, SampEn, $\alpha, \alpha_{\mathrm{S}}$ and $\alpha_{\mathrm{L}}$ ) over time among study subjects. For aim 2, we will use one-way analysis of variance to compare the BPV metrics between no-HTN, pre-HTN and HTN subjects. For aim 3, we will build a discriminant linear function of the variability indices in order to classify the subjects. Baseline data will be used for aims 2 and 3. Algorithms for computing entropies and the alpha exponents are implemented in MATLAB. All tests will be two-tailed and a $p<0.05$ will be considered significant. The statistical analyses will be conducted using STATA V.13 and SPSS V.24 for Windows.

\section{Patient and public involvement}

Patients and the public were not involved in the design of the study. Patients are recruited on a visit-to-visit basis. No research-specific visits are required. Study results will be made available to the public through publications in 
peer-reviewed journals and conference papers and/or presentations.

\section{Ethics and dissemination}

Approached subjects have an identification number (ID). Those who meet the inclusion criteria and are willing to participate have a documented name linked to their ID number and their name will appear on all consent forms. Documents with participants' names are kept in a locked drawer in the principal investigator's office. Only the principal investigator has access to that drawer. The study protocol is approved by the Institutional Review Board (IRB) at the American University of Beirut (IRB ID: BIO2018-0040). Additionally, the research team members are certified by the Collaborative Institutional Training Initiative (CITI).

Results of the study are going to be submitted for publication in peer-reviewed, refereed scientific journals and presented in national and international conferences. Additionally, the results will be shared at the Vascular Medicine Programme at AUBMC to promote the importance and significance of beat-to-beat BPV profile characterisation among patients.

\section{Study status at the time of manuscript submission}

The subjects' recruitment started in October 2019 and was delayed due to several reasons including COVID-19 lockdown and civil unrest in the country. After initiation of the study and before the lockdown, we recruited 11 patients preoperative and 2 patients at 3 and 6 months postoperative follow-up visits. The average age of the first seven recruited subjects is 42.43 years, and the average BMI is 41.07. Three subjects were females, 5 on HTN medication and all subjects had comorbidities; BPV parameters of these subjects are presented in table 1 .

\section{DISCUSSION}

Previous studies investigated the effect of autonomic control on beat-to-beat BPV $^{213233}$ and showed that an increased sympathetic activity could result in changes in BPV related to increased vascular resistance. ${ }^{32}$ This is particularly important in obese individuals who are known to have altered sympathovagal balance. ${ }^{34}$ In fact, the latter has been shown to be a determinant of shortterm variability. ${ }^{35}$ While the status of HR variability in obese individuals has been previously described, few studies characterise changes in beat-to-beat BPV, particularly in terms of complexity and self-correlation of BP signal in obese and morbidly obese subjects. Additionally, to the best of our knowledge, the responsiveness of altered BP dynamics, that is, variability, to surgical weight loss has not been previously assessed.

In our study, we expect to observe altered BPV profiles, reflected by increased linear fluctuations and self-correlation as well as decreased complexity of beatto-beat BP time series, in HTN and pre-HTN individuals as compared with their normotensive counterparts. We also expect that BPV profiles will improve with weight loss after bariatric surgery, although differentially across the different HTN categories. Our study is also speculated to establish a relationship between beat-to-beat BPV, body weight and average BP.

We acknowledge the fact that in the obese hypertensive group, antihypertensive treatment regimens might differentially influence beat-to-beat dynamics. ${ }^{36} 37$ Some drug classes were associated with positive outcomes with respect to control BPV; while others had negative outcomes on variability. ${ }^{38}$ However, such results were derived from intermittent BP monitoring and thus, unlike our present study, variability measures were calculated based on

\begin{tabular}{|c|c|c|c|c|c|c|c|c|}
\hline Subject & Series & $\begin{array}{l}\text { Average } \\
\text { (Brachial) }\end{array}$ & No of beats & Linear & & Non-linea & & \\
\hline & & & & SD & $\mathrm{CV}$ & SampEn & ApEn & DFA $\alpha$ \\
\hline 1 & $\begin{array}{l}\text { SBP } \\
\text { DBP }\end{array}$ & $\begin{array}{l}155 \\
98\end{array}$ & 1620 & $\begin{array}{l}11.56 \\
7.61\end{array}$ & $\begin{array}{l}7.74 \\
9.69\end{array}$ & $\begin{array}{l}0.95 \\
1.02\end{array}$ & $\begin{array}{l}0.98 \\
1.09\end{array}$ & $\begin{array}{l}0.93 \\
0.98\end{array}$ \\
\hline 2 & $\begin{array}{l}\text { SBP } \\
\text { DBP }\end{array}$ & $\begin{array}{l}130 \\
91\end{array}$ & 1792 & $\begin{array}{l}5.81 \\
6.43\end{array}$ & $\begin{array}{l}4.38 \\
7.4\end{array}$ & $\begin{array}{l}1.45 \\
0.83\end{array}$ & $\begin{array}{l}1.38 \\
0.93\end{array}$ & $\begin{array}{l}0.75 \\
0.88\end{array}$ \\
\hline 3 & $\begin{array}{l}\text { SBP } \\
\text { DBP }\end{array}$ & $\begin{array}{l}160 \\
83\end{array}$ & 2722 & $\begin{array}{l}15.24 \\
8.74\end{array}$ & $\begin{array}{l}9.56 \\
9.95\end{array}$ & $\begin{array}{l}1.33 \\
1.38\end{array}$ & $\begin{array}{l}1.35 \\
1.36\end{array}$ & $\begin{array}{l}1.00 \\
1.07\end{array}$ \\
\hline 4 & $\begin{array}{l}\text { SBP } \\
\text { DBP }\end{array}$ & $\begin{array}{l}133 \\
75\end{array}$ & 2301 & $\begin{array}{l}21.19 \\
12.89\end{array}$ & $\begin{array}{l}14.11 \\
13.61\end{array}$ & $\begin{array}{l}0.37 \\
0.53\end{array}$ & $\begin{array}{l}0.43 \\
0.60\end{array}$ & $\begin{array}{l}1.12 \\
1.12\end{array}$ \\
\hline 5 & $\begin{array}{l}\text { SBP } \\
\text { DBP }\end{array}$ & $\begin{array}{l}139 \\
94\end{array}$ & 1994 & $\begin{array}{l}17.48 \\
12.47\end{array}$ & $\begin{array}{l}13.09 \\
13.96\end{array}$ & $\begin{array}{l}0.60 \\
0.71\end{array}$ & $\begin{array}{l}0.71 \\
0.83\end{array}$ & $\begin{array}{l}1.03 \\
0.97\end{array}$ \\
\hline 6 & $\begin{array}{l}\text { SBP } \\
\text { DBP }\end{array}$ & $\begin{array}{l}168 \\
78\end{array}$ & 1714 & $\begin{array}{l}16.62 \\
5.30\end{array}$ & $\begin{array}{l}10.95 \\
7.48\end{array}$ & $\begin{array}{l}1.08 \\
0.76\end{array}$ & $\begin{array}{l}1.03 \\
0.79\end{array}$ & $\begin{array}{l}1.07 \\
0.98\end{array}$ \\
\hline 7 & $\begin{array}{l}\text { SBP } \\
\text { DBP }\end{array}$ & $\begin{array}{l}156 \\
98\end{array}$ & 2155 & $\begin{array}{l}8.48 \\
5.29\end{array}$ & $\begin{array}{l}6.12 \\
6.13\end{array}$ & $\begin{array}{l}1.35 \\
0.82\end{array}$ & $\begin{array}{l}1.25 \\
0.90\end{array}$ & $\begin{array}{l}0.94 \\
0.85\end{array}$ \\
\hline
\end{tabular}

ApEn, approximate entropy; BPV, blood pressure variability; CV, coefficient of variation; DBP, diastolic blood pressure; DFA, detrended fluctuation analysis; SampEn, sample entropy; SBP, systolic blood pressure. 
non-continuous BP. We, therefore, aim at soliciting drug therapy-related information from patient charts.

Two additional factors are worth inspecting and are indeed taken into account. Those are gender differences and family history of cardiovascular disease. Indeed, offspring of hypertensive parents were found to present with an altered profile of beat-to-beat BPV in response to an autonomic challenge, like a cold pressor test, when compared with offspring of normotensive parents. ${ }^{22}$ Finally, gender-specific differences in adaptation to metabolic and cardiovascular insults and responsiveness to abrupt ameliorative interventions are worth discovering especially in light of previous studies. ${ }^{39} 40$

Our study will be the first to look at fairly novel measures of BPV among obese and morbidly obese subjects undergoing bariatric surgery and their relationship with body weight and average BP. Application of continuous, non-invasive BP monitoring for characterisation of BP complexity and self-correlation may be helpful in providing insight into the early deteriorating mechanisms of cardiovascular control.

\section{Twitter Nour-Mounira Z Bakkar @NBakkar_94}

Contributors Conceptualisation, SF and KB; methodology, SF, N-MZB and RA; software, SF; validation, SF and N-MZB; formal analysis, SF, N-MZB, RA, IL and KB; investigation, SF, N-MZB, RA, IL and KB; data curation, SF and N-MZB; writingoriginal draft preparation, SF and N-MZB; writing - review and editing, SF, N-MZB, IL and KB; visualisation: SF; supervision: SF; project administration: SF; funding acquisition: SF. All authors have read and agreed to the published version of the manuscript.

Funding This work is supported by funds to SF from the American University of Beirut, Faculty of Medicine, Medical Practice Plan, Grant \#320162.

Disclaimer The funding body approved the design of the study. However, it has no role in data collection, analysis or interpretation or in manuscript writing. The funding body oversees the progress of the study and its contributions in terms of publications.

Competing interests None declared.

Patient and public involvement Patients and/or the public were not involved in the design, or conduct, or reporting, or dissemination plans of this research.

Patient consent for publication Consent obtained directly from patient(s)

Provenance and peer review Not commissioned; externally peer reviewed.

Open access This is an open access article distributed in accordance with the Creative Commons Attribution Non Commercial (CC BY-NC 4.0) license, which permits others to distribute, remix, adapt, build upon this work non-commercially, and license their derivative works on different terms, provided the original work is properly cited, appropriate credit is given, any changes made indicated, and the use is non-commercial. See: http://creativecommons.org/licenses/by-nc/4.0/.

\section{ORCID iD}

Nour-Mounira Z Bakkar http://orcid.org/0000-0002-6403-9348

\section{REFERENCES}

1 Khan SS, Ning H, Wilkins JT, et al. Association of body mass index with lifetime risk of cardiovascular disease and compression of morbidity. JAMA Cardiol 2018;3:280-7.

2 Lakoski SG, Cushman M, Siscovick DS, et al. The relationship between inflammation, obesity and risk for hypertension in the multi-ethnic study of atherosclerosis (MESA). J Hum Hypertens 2011;25:73-9.

3 Marini S, Merino J, Montgomery BE, et al. Mendelian randomization study of obesity and cerebrovascular disease. Ann Neurol 2020;87:516-24.
4 Strazzullo P, D'Elia L, Cairella G, et al. Excess body weight and incidence of stroke: meta-analysis of prospective studies with 2 million participants. Stroke 2010;41:e418-26.

5 Wolk R, Shamsuzzaman ASM, Somers VK. Obesity, sleep apnea, and hypertension. Hypertension 2003;42:1067-74.

6 Haines KL, Nelson LG, Gonzalez R, et al. Objective evidence that bariatric surgery improves obesity-related obstructive sleep apnea. Surgery 2007;141:354-8.

$7 \mathrm{Yu}$ J, Zhou X, Li L, et al. The long-term effects of bariatric surgery for type 2 diabetes: systematic review and meta-analysis of randomized and non-randomized evidence. Obes Surg 2015;25:143-58.

8 Liang Z, Wu Q, Chen B, et al. Effect of laparoscopic Rouxen-Y gastric bypass surgery on type 2 diabetes mellitus with hypertension: a randomized controlled trial. Diabetes Res Clin Pract 2013;101:50-6.

9 Algahim MF, Lux TR, Leichman JG, et al. Progressive regression of left ventricular hypertrophy two years after bariatric surgery. Am J Med 2010;123:549-55.

10 Buchwald $\mathrm{H}$, Avidor $\mathrm{Y}$, Braunwald E, et al. Bariatric surgery: a systematic review and meta-analysis. JAMA 2004;292:1724-37.

11 Parati G, Ochoa JE, Lombardi C, et al. Assessment and management of blood-pressure variability. Nat Rev Cardiol 2013;10:143-55.

12 Peng CK, Havlin S, Stanley HE, et al. Quantification of scaling exponents and crossover phenomena in nonstationary heartbeat time series. Chaos 1995:5:82-7.

13 Pincus SM, Goldberger AL. Physiological time-series analysis: what does regularity quantify? Am J Physiol 1994;266:H1643-56.

14 Subramaniam B, Khabbaz KR, Heldt T, et al. Blood pressure variability: can nonlinear dynamics enhance risk assessment during cardiovascular surgery? J Cardiothorac Vasc Anesth 2014;28:392-7.

15 Rangasamy V, Henriques TS, Xu X, et al. Preoperative blood pressure complexity indices as a marker for frailty in patients undergoing cardiac surgery. J Cardiothorac Vasc Anesth 2020;34:616-21.

16 Trunkvalterova Z, Javorka M, Tonhajzerova I, et al. Reduced shortterm complexity of heart rate and blood pressure dynamics in patients with diabetes mellitus type 1: multiscale entropy analysis. Physiol Meas 2008;29:817-28.

17 Angelini L, Maestri R, Marinazzo D, et al. Multiscale analysis of short term heart beat interval, arterial blood pressure, and instantaneous lung volume time series. Artif Intell Med 2007;41:237-50.

18 Xia Y, Liu X, Wu D, et al. Influence of beat-to-beat blood pressure variability on vascular elasticity in hypertensive population. Sci Rep 2017;7:8394.

19 Wei F-F, Li Y, Zhang L, et al. Beat-to-beat, reading-to-reading, and day-to-day blood pressure variability in relation to organ damage in untreated Chinese. Hypertension 2014;63:790-6.

20 Webb AJS, Mazzucco S, Li L, et al. Prognostic significance of blood pressure variability on beat-to-beat monitoring after transient ischemic attack and stroke. Stroke 2018;49:62-7.

21 Turianikova Z, Javorka K, Baumert M, et al. The effect of orthostatic stress on multiscale entropy of heart rate and blood pressure. Physiol Meas 2011;32:1425-37.

22 Wu D, Xu L, Abbott D, et al. Analysis of beat-to-beat blood pressure variability response to the cold pressor test in the offspring of hypertensive and normotensive parents. Hypertens Res 2017;40:581-9.

23 Bakkar N-MZ, El-Yazbi AF, Zouein FA, et al. Beat-to-beat blood pressure variability: an early predictor of disease and cardiovascular risk. J Hypertens 2021;39:830-45.

24 Javorka M, Turianikova Z, Tonhajzerova I, et al. Heart rate and blood pressure control in obesity-how to detect early dysregulation? Clin Physiol Funct Imaging 2016;36:337-45.

25 Latchman PL, Mathur M, Bartels MN, et al. Impaired autonomic function in normotensive obese children. Clin Auton Res 2011:21:319-23.

26 Avdeeva K, Petelina T, Gapon L, et al. Variability of blood pressure as a risk factor of increased rigidity of vascular wall in patients with arterial hypertension and abdominal obesity. Atherosclerosis 2017;263:e126

27 Nault I, Nadreau E, Paquet C, et al. Impact of bariatric surgeryinduced weight loss on heart rate variability. Metabolism 2007;56:1425-30.

28 Machado MB, Velasco IT, Scalabrini-Neto A. Gastric bypass and cardiac autonomic activity: influence of gender and age. Obes Surg 2009;19:332-8.

29 Cerutti S, Corino VDA, Mainardi L, et al. Non-Linear regularity of arterial blood pressure variability in patient with atrial fibrillation in tilt-test procedure. Europace 2014;16:iv141-7.

30 Zhang Y, Chan GSH, Tracy MB, et al. Detrended fluctuation analysis of blood pressure in preterm infants with intraventricular hemorrhage. Med Biol Eng Comput 2013;51:1051-7. 
31 Fares SA, Habib JR, Engoren MC, et al. Effect of salt intake on beat-to-beat blood pressure nonlinear dynamics and entropy in salt-sensitive versus salt-protected rats. Physiol Rep 2016;4:e12823.

32 Xia Y, Wu D, Gao Z, et al. Association between beat-to-beat blood pressure variability and vascular elasticity in normal young adults during the cold pressor test. Medicine 2017;96:e6000.

33 Reulecke S, Charleston-Villalobos S, Voss A, et al. Orthostatic stress causes immediately increased blood pressure variability in women with vasovagal syncope. Comput Methods Programs Biomed 2016;127:185-96.

34 Indumathy J, Pal GK, Pal P, et al. Decreased baroreflex sensitivity is linked to sympathovagal imbalance, body fat mass and altered cardiometabolic profile in pre-obesity and obesity. Metabolism 2015:64:1704-14.

35 Laitinen T, Hartikainen J, Niskanen L, et al. Sympathovagal balance is major determinant of short-term blood pressure variability in healthy subjects. Am J Physiol 1999;276:H1245-52.
36 Bertera FM, Del Mauro JS, Lovera V, et al. Acute effects of third generation $\beta$-blockers on short-term and beat-to-beat blood pressure variability in sinoaortic-denervated rats. Hypertens Res 2013;36:349-55

37 Frattola A, Parati G, Castiglioni P, et al. Lacidipine and blood pressure variability in diabetic hypertensive patients. Hypertension 2000;36:622-8.

38 Rothwell PM, Howard SC, Dolan E, et al. Effects of beta blockers and calcium-channel blockers on within-individual variability in blood pressure and risk of stroke. Lancet Neurol 2010;9:469-80.

39 Castiglioni P, Lazzeroni D, Coruzzi P. Multifractal-multiscale analysis of cardiovascular signals: a DFA-based characterization of blood pressure and heart-rate complexity by gender. Complexity 2018;2018.

40 Milan-Mattos JC, Porta A, Perseguini NM, et al. Influence of age and gender on the phase and strength of the relation between heart period and systolic blood pressure spontaneous fluctuations. J Appl Physiol 2018;124:791-804. 